\title{
CARACTERIZACIÓN FISIOLÓGICA DEL FRUTO DE CHAMPA (Campomanesia lineatifolia Ruiz \& Pavón) DURANTE LA POSCOSECHA
}

\section{PHYSIOLOGICAL CHARACTERIZATION OF CHAMPA FRUIT (Campomanesia lineatifolia Ruiz \& Pavón) DURING POSTHARVEST}

\author{
Javier Giovanni Álvarez¹, Helber Enrique Balaguera², Julián Fernando Cárdenas³
}

\begin{abstract}
${ }^{1}$ Profesor asistente. Grupo de Investigaciones Agrícolas, Facultad de Ciencias Agropecuarias, Universidad Pedagógica y Tecnológica de Colombia. Av. Central del Norte km 0 vía Paipa. Tunja, Colombia. e-mail: jgalvarezh@gmail.com, ${ }^{2}$ I.A. Grupo de Investigaciones Agrícolas, Facultad de Ciencias Agropecuarias, Universidad Pedagógica y Tecnológica de Colombia. Av. Central del Norte km 0 vía Paipa. Tunja, Colombia. e-mail: enrique_balaguera@yahoo.com, ${ }^{3}$ I.A. Grupo de Investigaciones Agrícolas, Facultad de Ciencias Agropecuarias, Universidad Pedagógica y Tecnológica de Colombia. Av. Central del Norte km 0 vía Paipa Tunja, Colombia. e-mail: julianentero@gmail.com
\end{abstract}

Rev. U.D.C.A Act. E Div. Cient. 12 (2): 125-133, 2009

\section{RESUMEN}

El fruto de champa posee características organolépticas que lo hacen agradable al consumidor. Sin embargo, es una fruta poco conocida y a la cual, no se le ha determinado el comportamiento poscosecha. Por lo anterior, se llevó a cabo el estudio de los cambios fisicoquímicos de los frutos de champa, a los que se les determinó diariamente la tasa respiratoria, la firmeza, el pH, los sólidos solubles totales (SST), la acidez total titulable (ATT), el índice de madurez (IM) y la pérdida de masa en la fase de poscosecha. Se observó un comportamiento climatérico en la respiración de la champa. El período poscosecha a condiciones de temperatura ambiente $\left(18^{\circ} \mathrm{C}\right.$ y $75 \%$ de humedad relativa) fue de cuatro días, que indicó que la champa es un fruto altamente perecedero. La firmeza presentó una disminución lineal al pasar de 3,2N a $1 \mathrm{~N}$. El pH, los SST y el IM aumentaron hasta el segundo día, luego mostraron un descenso hasta el tercer día, para finalmente estabilizarse, mientras que la ATT tuvo una leve disminución en el segundo día, aumentó para el tercero, donde se logró mantener constante. La pérdida de masa aumentó linealmente durante el periodo poscosecha, con un valor promedio de 2,35\% por día.
Palabras clave: Intensidad respiratoria, firmeza, sólidos solubles, acidez titulable, frutales tropicales.

\section{SUMMARY}

The champa fruit has organoleptic characteristics that makes it pleasant to the consumer. However, it is a littleknown fruit, for which post-harvest behavior has not been established.. Therefore, a study of the physicochemical changes of the champa fruit was carried out, in which the daily the respiratory rate, firmness, $\mathrm{pH}$, total soluble solids (TSS), the total titratable acidity (TTA), the maturity index (MI) and the loss of mass in the post-harvest phase was determined. Climacteric behavior was observed in the champa fruit respiration. The postharvest period, at ambient temperature $\left(18^{\circ} \mathrm{C}\right.$ y $75 \%$ of relative humidity) condition, was four days, which indicated that the champa is a highly perishable fruit. The firmness revealed a linear decrease passing from $3,2 \mathrm{~N}$ to $1 \mathrm{~N}$. The $\mathrm{pH}$, the TSS and the MI increased up to the second day, and then showed a decline until the third day, to eventually stabilize, while the TTA presented a slight decrease at the second day, increased for the third day one, staying constant from than on. The loss of mass increased 
linearly during the postharvest period with a mean value of $2.35 \%$ per day.

Key words: Respiratory rate, firmness, soluble solids, titratable acidity, tropical fruits.

\section{INTRODUCCIÓN}

La champa es un árbol frutal originario de la Amazonía (Villachica, 1996), que pertenece a la familia Myrtaceae (Bonilla et al. 2005), descrita por Ruiz y Pavón en 1798, encontrando evidencias de frutos de champa en excavaciones realizadas a vestigios de ciudades de la cultura Nazca (Piacenza, 2005). Crece en condiciones de temperaturas de 22 a $30^{\circ} \mathrm{C}$, con precipitaciones superiores a $1.500 \mathrm{~mm}$ anuales y necesita suelos de texturas francas a arcillosas (Villachica, 1996). El fruto es una baya ligeramente achatada de hasta $7 \mathrm{~cm}$ de diámetro, pesa en promedio $22 \mathrm{~g}$ (López $\mathcal{E}$ Rodríguez, 1995); la corteza es amarilla en estado maduro y difícilmente separable de la pulpa; el ápice es truncado, coronado y con cáliz persistente. La pulpa es carnosa con cuatro a diez semillas aplanadas y circulares, de $1 \mathrm{~cm}$ de diámetro, color marrón claro y de sabor amargo (Villachica, 1996).

La cosecha de los frutos, se inicia aproximadamente cuatro meses después de la floración, entre agosto y octubre, en el municipio de Miraflores, en el departamento de Boyacá, el cual, es el mayor productor de este fruto en Colombia. En estado de máxima producción, un árbol puede producir hasta $500 \mathrm{~kg}$ de fruta por año. Debido a la altura de los árboles, la cosecha se dificulta, por lo que, generalmente, se espera que los frutos maduren y caigan para hacer la recolección (López \& Rodríguez, 1995).

El particular sabor y aroma del fruto son características agradables al consumidor. Las $\beta$-tricetonas contribuyen a expresar el aroma característico de la champa (Osorio et al. 2006) y se denominan champanones A, B y C, cuya actividad es antibacterial (Bonilla et al. 2005). Además, es un fruto altamente perecedero, lo que limita su comercialización en fresco y su presencia en el mercado se ve reducida a las zonas de producción y a la venta en forma de pulpa (Villachica, 1996).

Durante la poscosecha, se deben considerar los cambios que se producen en los frutos. Una vez éstos se recolectan, el metabolismo cambia, ya que se interrumpe el suministro de agua y de nutrientes, se detiene el proceso de fotosíntesis, se altera la atmósfera interna de los tejidos y también la composición de la atmósfera que rodea al fruto, debido a su propia respiración (Kays, 2004).

Por lo anterior, el objetivo de esta investigación fue realizar la caracterización fisiológica de los frutos de champa durante la poscosecha, bajo condiciones ambientales de $18^{\circ} \mathrm{C}$ y $75 \%$ de humedad relativa.

\section{MATERIALES Y MÉTODOS}

Los frutos de champa utilizados en el estudio se cosecharon manualmente en estado de madurez de consumo (60\% amarillo - $40 \%$ verde claro), con un tamaño homogéneo y libres de mosca de la fruta (Anastrepha striata), reportada por Carrejo \& González (1999), principal limitante de la calidad de los frutos. Estos provenían de árboles de aproximadamente 15 años de edad, de las fincas "El mango" y "La playa" de la vereda Ayatá, del municipio de Miraflores (Boyacá), ubicado a $1432 \mathrm{msnm}$, latitud $5^{\circ} 11^{\prime}$ y longitud $73^{\circ} 08^{\prime}$, el cual, presenta una precipitación promedio de $2500 \mathrm{~mm}$ y una temperatura que oscila entre los 18 y $24^{\circ} \mathrm{C}$. Las mediciones de las variables poscosecha, se realizaron en el laboratorio de Ingeniería Agrícola de la Universidad Nacional de Colombia sede Bogotá, que presentó una temperatura ambiente promedio de $18^{\circ} \mathrm{C}$ y $75 \%$ de humedad relativa.

Se escogieron 80 frutos de aproximadamente el mismo peso $(25,6 \pm 1,22 \mathrm{~g})$ y se utilizaron 20 frutos diarios durante cuatro días para medir firmeza, mediante un penetrómetro Bertuzzi Fruit Tester FT327; SST, en porcentaje con un refractómetro Carl Zeiss; el pH, se determinó con un potenciómetro Orión modelo 420; la ATT, mediante la titulación con $\mathrm{NaOH} 0,1 \mathrm{~N}$ hasta $\mathrm{pH} 8,2$ y se expresó como ácido málico y, finalmente, el índice de madurez (IM), se determinó como la relación SST/ ATT. Debido a que los frutos son altamente perecederos solo fue posible realizar mediciones durante cuatro días después de la cosecha, ya que estos presentaron pudrición, ablandamiento, ataque de hongos y oxidación de tejidos.

La intensidad respiratoria $\left(\mathrm{mg} \cdot \mathrm{CO}_{2} \cdot \mathrm{h}^{-1} \cdot \mathrm{kg}^{-1}\right)$, se estimó por el método de Petenkoffer, descrito por Villamizar 
(2001). Se tomaron 500g de fruta (aproximadamente, 20 frutos) y se introdujeron en un recipiente hermético; luego, el producto de la respiración fue neutralizado con $\mathrm{NaOH}(0,1 \mathrm{~N})$. La cantidad de $\mathrm{H}_{2} \mathrm{CO}_{3}$ producido, se determinó a partir de la diferencia de concentraciones de la solución de $\mathrm{NaOH}(0,1 \mathrm{~N})$ inicial y la concentración de $\mathrm{NaOH}$ parcialmente neutralizada. A partir del $\mathrm{H}_{2} \mathrm{CO}_{3}$, por estequiometria, se obtuvo la cantidad de $\mathrm{CO}_{2}$. La determinación de la intensidad respiratoria, se llevó a cabo con tres repeticiones simultáneas por día durante el periodo de medición. Se determinó la pérdida de masa diaria con respecto a la masa inicial, mediante una balanza Acculab VIC 612 de 0,01g de precisión.

Para las diferentes variables medidas, se efectuó un análisis descriptivo, en el cual, se determinó el promedio y la desviación estándar. Del mismo modo, se ajustaron modelos estadísticos al comportamiento de los parámetros medidos y se aplicó la prueba de correlaciones de Pearson entre variables, con el software SAS v. 8.1e (Cary, N.C).

\section{RESULTADOS Y DISCUSIÓN}

La tasa respiratoria en los frutos de champa mostró un comportamiento climatérico, que se caracterizó por un descenso en la tasa de producción de $\mathrm{CO}_{2}$ durante los tres primeros días, con un posterior incremento en la respiración hasta alcanzar un punto máximo (pico climatérico) en el cuarto día, para luego descender nuevamente (Figura 1), lo que determinó la senescencia del fruto, comportamiento similar al descrito por Kader (2002), para frutos climatéricos. La respiración climatérica esta presente también en otras especies de la familia Myrtaceae, como la feijoa (Acca sellowiana O. Berg Burret) (Galvis, 2003; Valderrama et al. 2005), el guayabo (Psidium guajava) (Laguado et al. 1999) y el arazá (Eugenia stipitata Mc. Vaugh) (Hernández et al. 2007).

Los resultados indican que el preclimaterio de los frutos a temperatura ambiente, se obtuvo a los tres días y se alcanzó el pico climatérico $\left(38,39 \mathrm{mg} \cdot \mathrm{CO}_{2} \cdot \mathrm{Kg}^{-1} \cdot \mathrm{h}^{-1}\right)$ a los cuatro días después de la cosecha (ddc). La champa muestra un comportamiento semejante al arazá, que alcanza el climaterio entre el tercer y el quinto día (Hernández et al. 2007). Dicho comportamiento sugiere una vida poscosecha corta (Bower et al. 1998).

Para el arazá, el máximo climatérico del fruto coincide con una máxima producción de etileno de $10 \mu \mathrm{L} \cdot \mathrm{kg}^{-1} \cdot \mathrm{h}^{-1}$, comportamiento que es muy similar con el de guayaba (Hernández et al. 2007) y que, debido a la similitud en el comportamiento poscosecha, posiblemente también lo sea para la champa.

La pérdida de firmeza durante la poscosecha del fruto de champa presentó un comportamiento lineal con una alta pendiente (Figura 2), ya que inicia con un valor

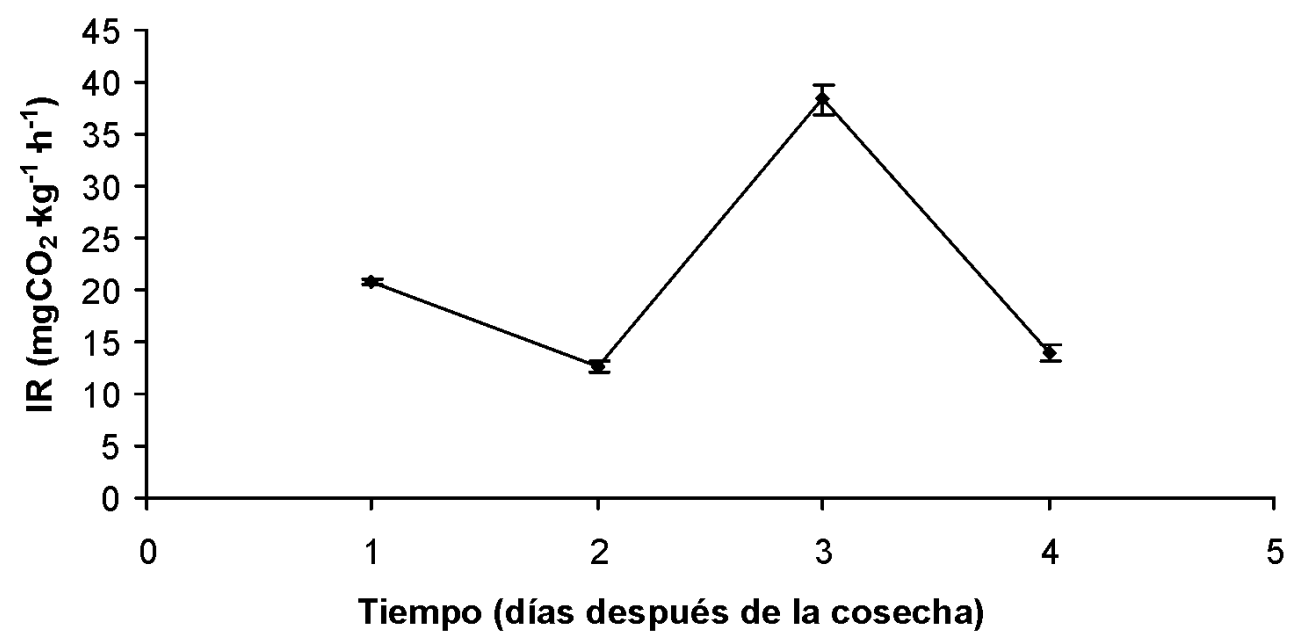

Figura 1. Variación de la intensidad respiratoria en la poscosecha del fruto de champa. La barra vertical indica la desviación estándar $(n=3)$. 
cercano a los 3,2N y termina en $1 \mathrm{~N}$. Estos valores son muy inferiores a los encontrados en frutas como el arazá (35N a 20N) (Hernández et al. 2007).

La carga que puede soportar un fruto equivale al $70 \%$ de su resistencia (Márquez et al. 2007), por lo tanto, frutos de champa durante los tres primeros ddc pueden soportar cargas cercanas a los $2 \mathrm{~N}$ sin que se vean afectados; después de este periodo, una carga mayor a $1 \mathrm{~N}$ causaría deterioro en la calidad. Estos valores de resistencia indican la susceptibilidad de la champa a daños físicos y dificultan las operaciones de acondicionamiento, lo que limita su comercialización en fresco, tal como lo afirman López \& Rodríguez (1995). La pérdida de firmeza en el fruto de champa es consecuencia de la degradación de la estructura de la pared celular y, en especial, de las alteraciones en el turgor de la célula. Esto se debe a la hidrólisis de la protopectina en fracciones más pequeñas e hidrosolubles (ácidos pécticos), que contribuyen al ablandamiento durante el proceso de maduración. El ablandamiento se convierte, entonces, en un factor limitante de comercialización (Kays, 2004; Kader, 2002).

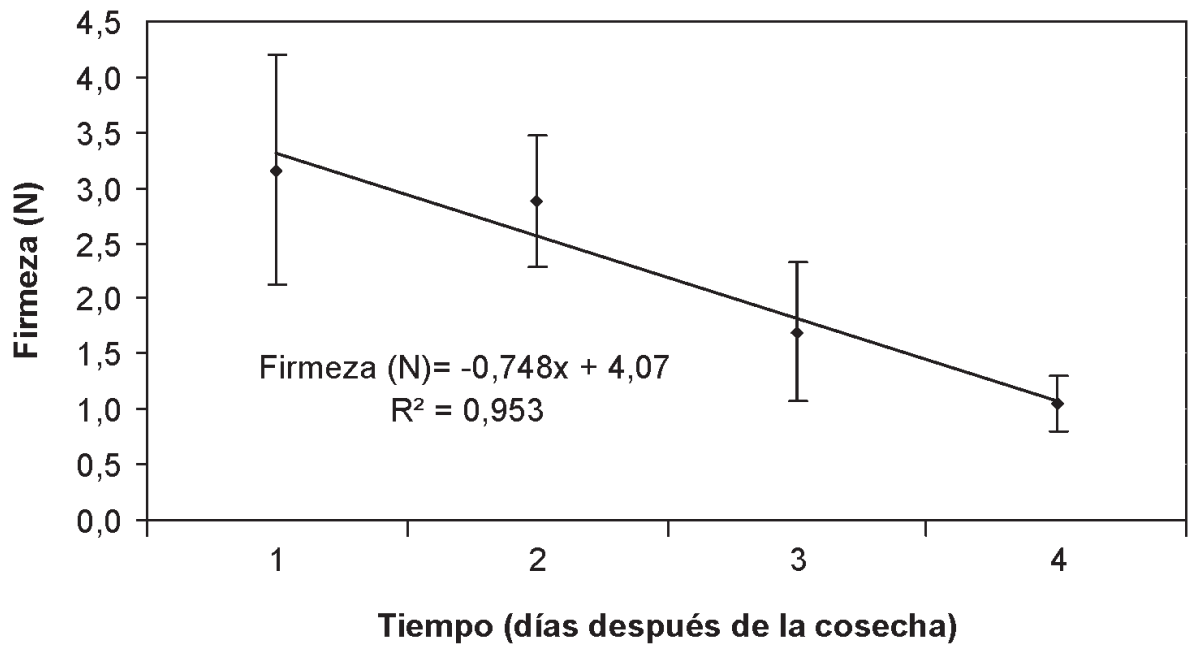

Figura 2. Variación de la firmeza en la poscosecha del fruto de champa. Las barras verticales indican la desviación estándar ( $\mathrm{n}=20)$.

La firmeza y la intensidad respiratoria no presentaron correlación (Tabla 1). No obstante, al incrementarse la intensidad respiratoria, se debe aumentar la pérdida de firmeza, porque al haber mayor respiración la concentración de etileno es más alta, lo cual, promueve la activación de enzimas, que intervienen en la degradación de la pared celular, lo que ocasiona el ablandamiento (Saladié et al. 2007); este proceso fue observado en frutos de Actinidia chinensis (Park et al. 2006), en donde hubo disminución de la firmeza conforme la respiración aumentó.

En las Figuras 3 y 4, se presenta el comportamiento del pH, la ATT, los SST, y el IM de frutos de champa en poscosecha. Se puede apreciar que los cambios observados en el $\mathrm{pH}$ fueron leves, se obtuvo un valor máximo de 2,89 en el tercer día y un valor mínimo de
2,62 en el cuarto, valores inferiores a los reportados para Campomanesia phae $(\mathrm{pH}=2,91)$, por Vallilo et al. (2005), quienes también afirman que a mayor acidez existe una mayor posibilidad de industrialización de los frutos en forma de dulces. Del mismo modo, Andrade et al. (1993) mencionan que para la elaboración de jugos, un alto nivel de acidez provoca una elevada dilución del producto $\mathrm{y}$, por consiguiente, un aumento en el rendimiento final del jugo.

Los SST oscilaron entre $11,2 \%$ y $13,4 \%$, valores muy superiores a los reportados para C. phae (Vallilo et al. 2005), E. klotzschiana (Vallilo et al. 2003), Myrciaria trunciflora Berg., E. uniflora L. y muy similares a los encontrados en P. guajava L. (Lajolo, 2001) y A. deliciosa (Manolopoulou \& Papadopoulou, 1998), por lo que se puede decir que la champa es un fruto dulce. La ATT 
Tabla 1. Correlaciones de Pearson para las variables poscosecha de los frutos de champa.

\begin{tabular}{|c|c|c|c|c|c|c|c|}
\hline & Intensidad respiratoria & Firmeza & Pérdida de masa & pH & SST & ATT & IM \\
\hline Intensidad respiratoria & 1 & & & & & & \\
\hline Firmeza & $-0,15 \mathrm{~ns}$ & 1 & & & & & \\
\hline Pérdida de masa & $0,08 \mathrm{~ns}$ & $-0,78^{* *}$ & 1 & & & & \\
\hline $\mathrm{pH}$ & $-0,71^{* *}$ & $0,53 \mathrm{~ns}$ & $-0,47 \mathrm{~ns}$ & 1 & & & \\
\hline SST & $-0,42 \mathrm{~ns}$ & $0,18 \mathrm{~ns}$ & $-0,09 \mathrm{~ns}$ & $0,7^{* *}$ & 1 & & \\
\hline ATT & $0,46 \mathrm{~ns}$ & $-0,75^{* *}$ & $0,82^{* *}$ & $-0,84^{* *}$ & $-0,4 \mathrm{~ns}$ & 1 & \\
\hline $\mathrm{IM}$ & $-0,53 \mathrm{~ns}$ & $0,59^{*}$ & $-0,6^{*}$ & $0,94^{* *}$ & $0,75^{* *}$ & $-0,90^{* *}$ & 1 \\
\hline
\end{tabular}

*, **: Diferencias significativas $(P<0,05)$ y altamente significativas $(P<0,01)$, respectivamente;

ns: no hay diferencias estadísticas.

presentó valores entre 4,9\% y 3,5\%, lo cual, la hace bastante ácida frente a otras mirtáceas, como la Acca sellowiana Berg (2,37\% a 0,87\%) (Rodríguez et al. 2006) y P. araca (2\% a 0,9\%) (Lara et al. 2007). El IM varío de 2,87 a 3,85 , valores superiores a los reportados por Hernández \& Barrera (2004) para arazá, con promedios 2,3 y para $C$. phae que presentó una relación de madurez de 0,15 (Vallilo et al. 2005); no obstante, la champa presenta valores superiores en SST y en ATT, lo que la convierte en una fruta más dulce y más ácida que las especies mencionadas, característica que le da un sabor muy agradable.
El pH, los SST y el IM aumentaron hasta el segundo día, luego presentaron un descenso hasta el tercer día, para finalmente estabilizarse (Figura 3 y 4 ). Mientras que la ATT tuvo una leve disminución en el segundo día, aumentó para el tercer día, donde logró mantenerse constante (Figura 3). Este incremento ocurrido en los primeros dos días en el $\mathrm{pH}$ esta dado, principalmente, por la reducción de la ATT (Villamizar, 2001), originada por la disminución de los $\mathrm{H}^{+}$a nivel vacuolar, los cuales, pasan a conformar esqueletos de azúcares y por el incremento en la actividad de las deshidrogenasas, que favorecen la conversión de ácidos orgánicos a azúcares,

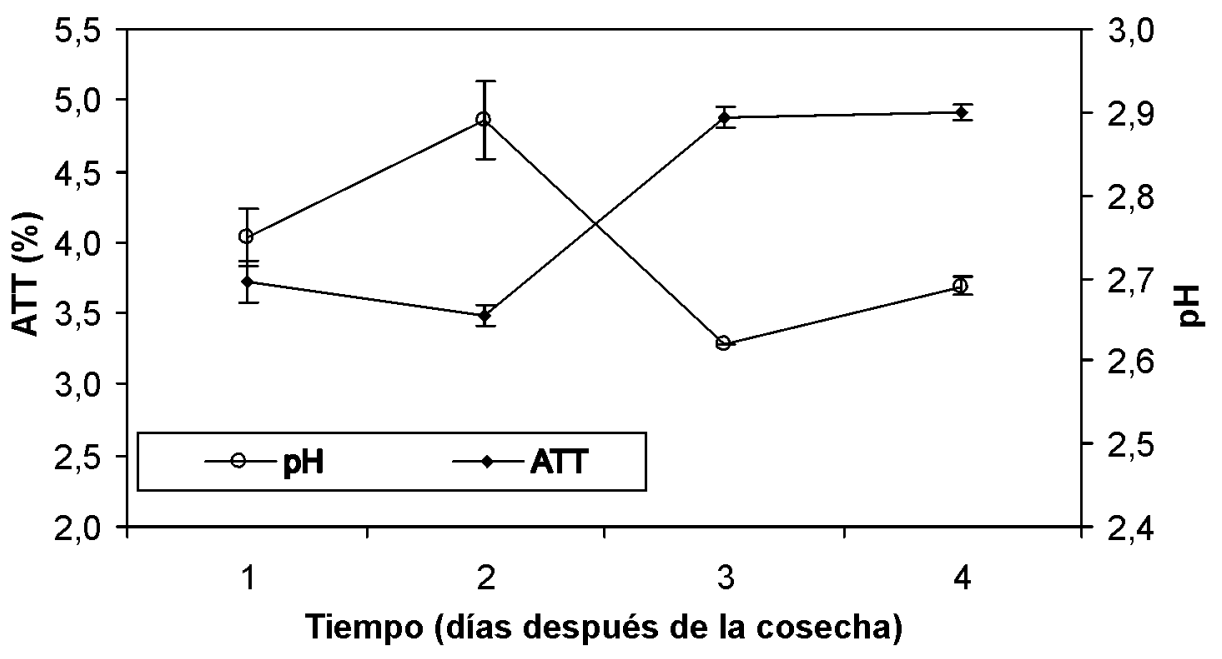

Figura 3. Comportamiento del pH y la ATT en la poscosecha de frutos de champa. Las barras verticales indican la desviación estándar $(n=3)$. 


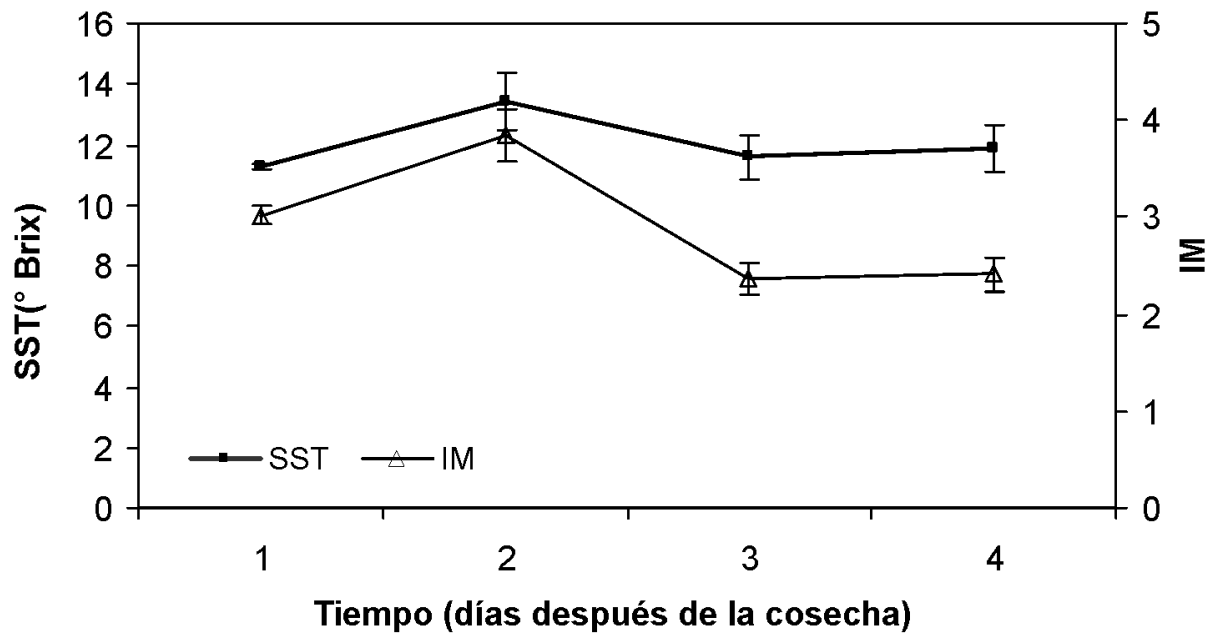

Figura 4. Comportamiento de los SST y el IM en la poscosecha de frutos de champa. Las barras verticales indican la desviación estándar ( $n=3)$.

utilizados como sustrato para la respiración (Marschner, 2002). Lo que coincide con el aumento que presentaron los SST hasta el día dos en los frutos de champa y con el respectivo aumento en el IM (Figura 4).

La disminución del $\mathrm{pH}$ y el aumento de la ATT después del día dos son atípicos al compararse con el comportamiento de estas dos variables en otros frutos, como la A. sellowiana Berg (Rodríguez et al. 2006) y P. guajava cv. "Media China" (Mercado-Silva et al. 1998). Este aumento en la ATT, probablemente, se puede dar porque al incrementarse el proceso de transpiración del fruto, la cantidad de agua en el fruto se hace menor y la concentración de ácidos orgánicos se aumenta, lo que se corrobora con la alta correlación existente entre estas dos variables (Tabla 1); además, es posible que los ácidos orgánicos producidos por la champa no sean primordialmente los utilizados en la respiración, si no que en ésta, se estén usando directamente los azúcares ya existentes (Mercado-Silva et al. 1998), razón por lo que disminuyeron los SST y de igual forma el IM.

Lo anterior sugiere que el fruto de champa posee unas reservas de almidón y de polisacáridos suficientes para cubrir la mayor parte de los requerimientos energéticos de las reacciones ocurridas en el climaterio y además pueden dar origen a ácidos grasos (Kays, 2004), lo cual, sumado a un posible aumento de los ácidos pécticos por destrucción de las paredes celulares (Wills et al. 1998), puede contribuir al aumento de la ATT, observado durante el climaterio. El aumento de la ATT también es reportado en banano (Cavendish Valery), por Zhang et al. (2005) y Ciro et al. (2005).

Todas las variables muestran un cambio de comportamiento el día anterior al climaterio y tienden a estabilizarse después del mismo. La prueba de correlaciones de Pearson determinó una relación directamente proporcional y significativa entre el $\mathrm{pH}$ con los SST y el IM, e inversamente con la ATT. Además, hubo una correlación directa y altamente significativa entre el IM y los SST, mientras que para el IM y la ATT fue inversa. Se observa una pérdida considerable de masa en los frutos de champa a lo largo de los cuatro días después de la cosecha. Durante este periodo, el aumento en la pérdida de masa fue lineal, con una constante de 2,35\% por día y con una disminución total de 1,77g por fruto (Figura 5). Esta pérdida presentó una correlación altamente significativa e inversa con la firmeza (Tabla 1).

La disminución de masa en los frutos cosechados, se debe a la pérdida de agua y sustratos, ocasionada por la respiración (Kader, 2002). La merma de agua, a su vez, está influenciada por otros aspectos, como el tamaño del fruto, ya que entre más pequeño, mayor será el área superficial por unidad de volumen expuesta y, en consecuencia, habrá mayor intercambio gaseoso entre el interior celular y la atmósfera (Laguado et al. 1999), razón por la cual, los frutos de champa tienen un alto potencial de pérdida de agua. 


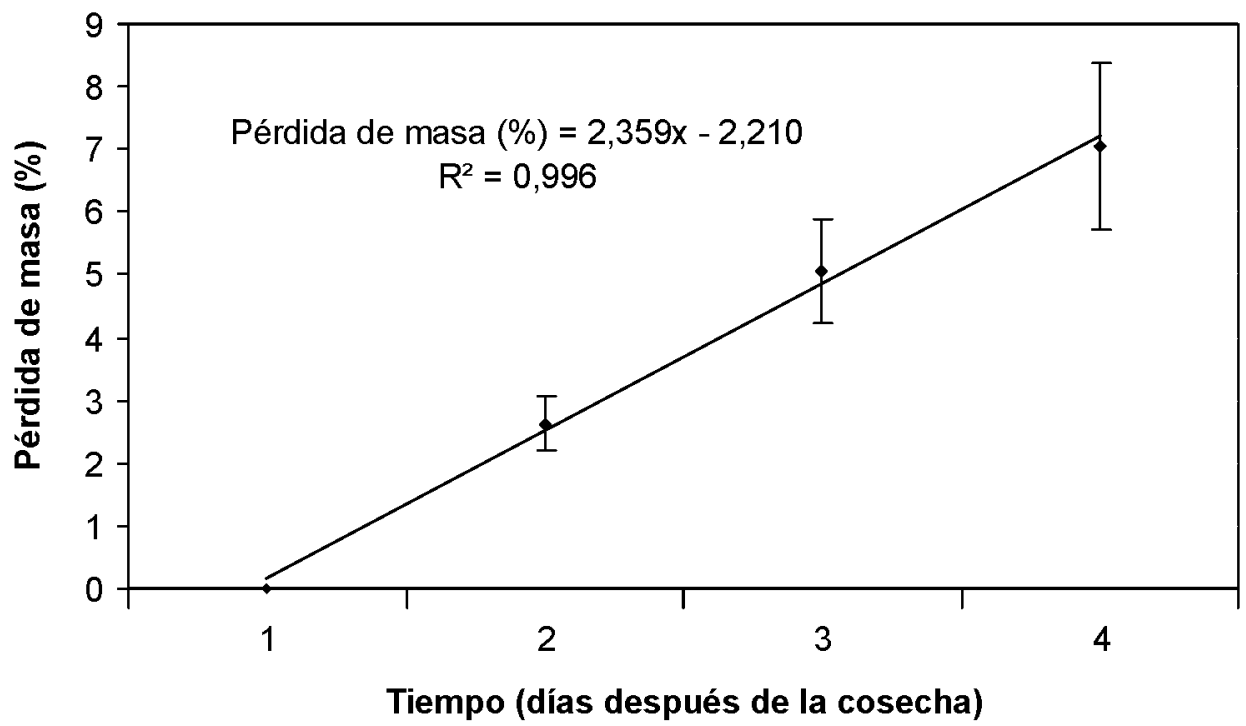

Figura 5. Pérdida de masa del fruto de champa en poscosecha. Las barras verticales indican la desviación estándar ( $n=3)$.

Como la pérdida total de masa durante los cuatro ddc fue de $6,82 \%$ y teniendo en cuenta que las disminuciones de masa superiores al $5 \%$ causan deterioro en la calidad del producto y, por consiguiente, reducción en el precio (Pantástico, 1981), entonces, el fruto de champa se debe comercializar antes de los cuatro ddc o someterlo a tratamientos poscosecha, que mantengan por más tiempo la calidad.

De los resultados obtenidos, se concluye que la champa es un fruto climatérico altamente perecedero y con valores muy bajos de firmeza en la poscosecha. La acidez de la champa, la hace una fruta viable para la elaboración de jugos. La ATT en los frutos durante la poscosecha presenta un comportamiento directamente proporcional con la pérdida de masa e inversamente proporcional con el $\mathrm{pH}$, los SST y el IM. La champa es una fruta dulce y ácida.

AGRADECIMIENTOS: Los autores expresan sus agradecimientos a María Inés López Martín, por su apoyo logístico y financiero al proyecto. Con su esmero colaboró en abrir la senda de la investigación sobre Champa en Colombia. Del mismo modo, agradecemos a los Ingenieros Rafael González Salinas y Miguel Ángel Díaz Herrera por su colaboración. Conflictos de intereses: Esta investigación y el manuscrito fueron realizados por todos los autores, por tanto, declaramos que no existe ningún conflicto de intereses que ponga en riesgo la validez de la presente publicación.

\section{BIBLIOGRAFÍA}

1. ANDRADE, J. de S.; ARAGÃO, C.G.; FERREIRA, S.A. do N. 1993. Caracterização física e química dos frutos de Araçá-Pêra (Psidium acutangulum D. C.). Acta Amazônica (Brasil). 23(2):213- 217.

2. BONILLA, A.; DUQUE, C.; GARZÓN, C.; TAKAISHI, Y.; YAMAGUCHI, K.; HARA, N.; FUJIMOTO, Y. 2005. Champanones, yellow pigments from the seeds of champa (Campomanesia lineatifolia). Phytochemistry (Reino Unido). 66:1736-1740.

3. BOWER, J.H.; JOBLING, J.J.; PATTERSON, B.D.; RYAN, D.J. 1998. A method for measuring the respiration rate and respiratory quotient of detached plant tissues. Postharvest Biol. Technol. (Holanda). 13:263-270.

4. CARREJO, N.; GONZÁLEZ, R. 1999. Parasitoids reared from species of Anastrepha (Diptera: Tephritidae) in Valle del Cauca, Colombia. Florida Entomologist. 82(1):113-118. 
5. CIRO, H.J.; MONTOYA, M.L.; MILLÁN L.J. 2005. Caracterización de propiedades mecánicas del banano (Cavendish Valery). Rev. Fac. Nal. Agr. Medellín (Colombia). 58(2):2975-2988.

6. GALVIS, A. 2003. Manejo de la cosecha y poscosecha de la feijoa. En: Fischer, G.; Miranda, D.; Cayón G.; Mazorra, M. eds. Cultivo, poscosecha y exportación de la feijoa (Acca selloviana Berg). Ed. Produmedios, (Bogotá). p.111-123.

7. HERNÁNDEZ, M.S.; BARRERA, J. 2004. Bases técnicas para el aprovechamiento agroindustrial de especies nativas de la Amazonía. Primera Edición. Ed. Guadalupe (Bogotá). 101p.

8. HERNÁNDEZ, M.S.; MARTÍNEZ, O.; FERNÁNDEZTRUJILLO, J.P. 2007. Behavior of arazá (Eugenia stipitata Mc Vaugh) fruit quality traits during growth, development and ripening. Sci. Hort. (Holanda). 111:220-227.

9. KADER, A.A. 2002. Standardization and inspection of fresh fruits and vegetables. En: Kader, A.A. ed. Postharvest technology of horticultural crops. $3^{\text {rd }}$ edition. Ed. University of California. Division of Agriculture and Natural Resources, (Oakland, CA). p.287-314.

10. KAYS, S. 2004. Postharvest biology. Ed. Exon Press (Athens, Georgia). 568p.

11. LAGUADO, N.; PÉREZ, E.; ALVARADO, C.; MARÍN, M. 1999. Características fisicoquímicas y fisiológicas de frutos de guayaba de los tipos Criolla Roja y San Miguel procedentes de dos plantaciones comerciales. Rev. Fac. Agron. (LUZ) (Venezuela). 16:382-397.

12. LAJOLO, M.F. 2001. Tabela brasileira de composição de alimentos. Disponible desde Internet en: www. fcf.usp.tabela. (con acceso 03/12/01).

13. LARA, C.; NERIO, L.S.; OVIEDO, L.E. 2007. Evaluación fisicoquímica y bromatológica de la guayaba agria (Psidium araca) en dos estados de maduración. Temas Agrarios (Colombia). 12(1):13-21.
14. LÓPEZ, M.; RODRÍGUEZ, J. 1995. Diagnóstico del mercadeo de la champa en el Municipio de Miraflores Boyacá. Trabajo de grado para optar al titulo de Tecnólogo en Mercadeo. Instituto de Educación Abierta y a Distancia. Universidad Pedagógica y Tecnológica de Colombia. Tunja. 86p.

15. MANOLOPOULOU, H.; PAPADOPOULOU, P. 1998. A study of respiratory and physico-chemical changes of four kiwifruit cultivars during cool-storage, Food Chemistry (Reino Unido). 63(4):529-534.

16. MÁRQUEZ, C.C.J.; OTERO, C.M.; CORTÉS, M. 2007. Cambios fisiológicos, texturales, fisicoquímicos y microestructurales del tomate de árbol (Cyphomandra betacea S.) en poscosecha. Vitae (Colombia). 14(2):9-16.

17. MARSCHNER, H. 2002. Mineral nutrition of higher plants. $2^{\text {nd }}$ edition. Ed. Academis Press (London). 889p.

18. MERCADO-SILVA, E.; BENITO-BAUTISTA, P.; GARCÍA-VELASCO, M. A. 1998. Fruit development, harvest index and ripening changes of guavas produced in central México. Postharvest Biol. Technol. 13:143-150.

19. OSORIO, C.; ALARCÓN, M.; MORENO, C.; BONILLA, A.; BARRIOS, J.; GARZÓN, C.; DUQUE, C. 2006. Characterization of Odor-Active Volatiles in Champa (Campomanesia lineatifolia R. \& P.). J. Agric. Food Chem. (EUA). 54(2):509-516.

20. PANTÁSTICO, E.R. 1981. Fisiología de Postrecolección. Vol.1. Ed. Limusa S.A. (México). p.812815.

21. PARK, Y.S.; JUNG, S.T.; GORINSTEIN, S. 2006. Ethylene treatment of 'Hayward' kiwifruits (Actinidia deliciosa) during ripening and its influence on ethylene biosintesis and antioxidant activity. Sci. Hort. 108:22-28.

22. PIACENZA, L. 2005. Evidencias botánicas en asentamientos Nasca. Boletín. Museo de Arqueología y Antropología (Peru). 5(1):3-13. 
23. RODRÍGUEZ, M.; ARJONA, H.; GALVIS, J. 2006. Maduración del fruto de feijoa (Acca sellowiana Berg) en los clones 41 (Quimba) y 8-4 a temperatura ambiente en condiciones de Bogotá. Agronomía Colombiana. 24(1):68-76.

24. SALADIÉ, M.; MATAS, A.J.; ISAACSON, T.; JENKS, M.A.; GOODWIN, S.M.; NIKLAS, K.J.; XIAOLIN, R.; LABAVITCH, J.M.; SHACKEL, K.A.; FERNIE, A.R.; LYTOVCHENKO, A.; O'NEILL, M.A.; WATKINS, C.B.; ROSE, J.K.C. 2007. A reevaluation of the key factors that influence tomato fruit softening and integrity. Plant Physiol. (EUA). 144:1012-1028.

25. VALDERRAMA, J.K.; FISCHER, G.; SERRANO, M.S. 2005. Fisiología poscosecha en frutos de dos cultivares de feijoa (Acca sellowiana O. Berg Burret) sometidos a un tratamiento. Agronomía Colombiana. 23(2):276-282.

26. VALLILO, M. I.; BAITELLO, J. B.; LAMARDO, L.; LOBANCO, C. M. 2003. Composição química do fruto de Eugenia klotzschiana Berg. (MYRTACEAE). Ver. Instituto Florestal (Brasil). 15(1):33-44.

27. VALLILO, M.I.; GARBELOTTI, M.L.; DE OLIVEIRA, E.; LAMARDO, L. 2005. Características físicas e químicas dos frutos do cambucizeiro (Campomanesia phaea). Rev. Bras. Frutic. 27(2):241-244.
28. VILLACHICA, H. 1996. Frutales y Hortalizas promisorios del Amazonas. Tratado de Cooperación Amazónica, Secretaría Pro Tempore (Lima). p.181-185.

29. VILLAMIZAR, F. 2001. Manejo tecnológico postcosecha de frutas y hortalizas. Aspectos Teóricos. Ed. Unidad de Publicaciones. Facultad de Ingeniería, Universidad Nacional de Colombia (Bogotá). 149p.

30. WILLS, R.; MCGLASSON, B.; GRAHAM, D.; JOYCE, D. 1998. Postharvest: An Introduction to the physiology and handling of fruit, vegetables and ornamentals. CABI Pub. p.97-112.

31. ZHANG, P.R.; WHISTLER, R.L.; BEMILLER J.N.; HAMAKER, B.R. 2005. Banana starch: production, physicochemical properties, and digestibility. A review. Carbohydrate Polymers (Reino Unido). 59:443-458.

Recibido: Diciembre 7 de 2008

Aceptado: Agosto 26 de 2009 